\title{
CT Perfusion Imaging: A Valuable and Feasible Resolution of Pulmonary Nodules
}

\author{
Mai-lin Chen, $\mathrm{MD}^{a}$, Ying-shi Sun, $\mathrm{MD}^{a, *}$ \\ ${ }^{a}$ Key Laboratory of Carcinogenesis and Translational Research (Ministry of Education/Beijing), Department of Radiology, Peking \\ University Cancer Hospital \& Institute, Beijing, China. \\ Received January 4, 2019; revision received February 21; accepted May 1.
}

\begin{abstract}
CT perfusion imaging, a non-invasive functional imaging method, can accurately reflect the hemodynamic changes of pulmonary nodules. It plays an important role in the diagnosis, differential diagnosis, treatment effect assessment and prognosis prediction. This article reviews the hemodynamics of pulmonary nodules, the characteristics of the blood flow of CT perfusion imaging of the pulmonary nodules, the characteristics of the CT perfusion technique of the pulmonary nodules and the status of the clinical application in the characterization of pulmonary nodules.
\end{abstract}

Key words: Pulmonary nodules; Hemodynamics; Perfusion imaging; Computed tomography

Advanced Ultrasound in Diagnosis and Therapy 2019;02:027-034

DOI: 10.37015/AUDT.2019.190806

$\mathrm{T}$ umor angiogenesis and development of antiangiogenic treatment regimens is a high priority area in cancer research. Reproducible and standardized biomarkers reflecting processes of tumor angiogenesis and assessing treatment effects of antiangiogenic tumor therapies are highly desirable $[1,2]$. For lung cancer, the hemodynamic change of pulmonary nodules is an important biomarker for evaluating the therapeutic effect as well [3]. Currently, significant efforts are being made to identify different biomarkers to characterize tumor perfusion using non-invasive technologies. For a long time, the level of molecular markers histologically measured, such as VEGF pathway, EGFR and its inhibitors have been used as the classic criteria in evaluating the tumor angiogenesis [3]. Dynamic contrast-enhanced ultrasound is not an ideal option for the assessment of lung tumors due to the limitations of ultrasound imaging in characterizing the nodules in air-filled lung, although it has been reported as a promising imaging tool for the depiction of tumor vasculature and perfusion in liver [4]. Dynamic contrastenhanced MRI has been proved helpful by numerous studies for the characterization of lung cancer perfusion and vascular permeability [5-9]. However, several issues regarding the technical aspects have to be addressed to achieve the correct perfusion quantification. Imaging biomarkers extracted from dynamic contrast-enhanced CT (DECT) or CT perfusion are based on the exchange of iodinated contrast agents between the intravascular space and the extravascular interstitial space. The main advantages of the technique include the easy integration in a routine clinical setting, ultrafast scans, linear relationship of iodine contrast concentrations and CT absorption numbers (Hounsfield Units, HU), reduced cardiac motion artifacts and increased perfusion coverage [10]. This review embraces the technical aspects of lung CT perfusion, the clinical applications of perfusion CT imaging in characterizing the hemodynamics of lung nodules, the value of perfusion $\mathrm{CT}$ in the diagnosis and differential diagnosis of pulmonary nodules and the evaluation of treatment effect, which is supposed to provide a comprehensive overview to familiarize the physicians with the technique of CT lung perfusion.

\section{CT Perfusion Techniques}

\section{Basic principles of CT perfusion imaging}

A fundamental principle of CT perfusion is the assessment of temporal changes in tissue attenuation after intravenous administration of iodinated contrast

\footnotetext{
* Corresponding Author: Key Laboratory of Carcinogenesis and Translational Research (Ministry of Education/Beijing), Department of Radiology, Peking University Cancer Hospital \& Institute, Beijing, China.

e-mail:sys27@163.com
} 
media. CT perfusion can be analyzed to quantify a range of physiological parameters that indicate the functional status of the vascular system within tumors and adjacent tissues. The attenuation, measured in Hounsfield units is linearly correlated with the concentration of contrast agent that passes the tumor vasculature. The parameters derived from two-compartment pharmacokinetics analysis of the dynamic change of contrast agent in tumors or nodules are comprised of intravascular and extravascular components.

Therefore, CT perfusion data for each subject is usually assessed in the form of quantitative maps of perfusion parameter by different mathematical models, the single- and dual-input maximum slope model and single-input Patlak plot model [11-13]. Several studies have indicated that the Patlak plot method is not well suitable for both dynamic CT perfusion data assessment for the differentiation diagnosis of pulmonary nodules, and therapeutic evaluation in patients with lung cancer [13] despite several vendors provide the software of Patlak plot model for quantitative assessment of dynamic CT perfusion data. Therefore, clinicians should gain a clear understanding of the mathematical models involved in these software products, when using dynamic CT perfusion examination in patients with pulmonary nodules.

\section{CT perfusion image acquisition protocols}

The first study that demonstrated the feasibility, the strengths and weaknesses of DECT in the work-up of solitary pulmonary nodules (SPNs) in clinical setting was published in 2008 [14]. After the introduction of multidetector row CT (MDCT) in clinical use, dynamic CT perfusion examination evolved from 2-4 detector CT to spectral 256 or 320-detector row CT. In current routine clinical practice, all dynamic CT perfusion studies can be performed using a dynamic volumetric scan, which obtains $160 \mathrm{~mm}$ volumetric thin-section CT data without helical scan $[11-13,15]$. Dynamic CT perfusion is generally obtained through the nodule with the technical aspects described in the following: (1) Intravenous contrast agent administration. Quantitative assessment modelling benefits from a bolus injection of contrast agent at an injection rate of at least $4 \mathrm{~mL} / \mathrm{s}$ (from $4 \mathrm{~mL} / \mathrm{s}$ to $8 \mathrm{~mL} / \mathrm{s}$ ) via a large bore intravenous cannula. The total injected iodine dose should be within the range of 12$18 \mathrm{~g}$ (about $20 \sim 60 \mathrm{~mL}$ of an iodinated contrast medium) and the iodine concentration administered is not less than $300 \mathrm{mg} / \mathrm{ml}$. For high viscosity contrast media with $>370$ $\mathrm{mg} / \mathrm{mL}$ iodine concentration, the contrast agent should be heated to body temperature to improve efficient bolus delivery. (2) CT perfusion data acquisition should be maintained for at least 30 s to achieve the initial perfusion phase, which is critical to ensure accurate quantification of regional blood flow, followed by $20 \mathrm{~mL}$ to $40 \mathrm{~mL}$ of saline solution at the same injection rate [11-15].

\section{Imaging analysis of CT perfusion parameters}

Semi-quantitative or quantitative CT perfusion parameters that reflect tumor vascularity are readily obtained from the time-attenuation curves of tumors by fitting the suitable mathematic model [16]. The calculation of CT perfusion parameters is performed using dedicated software. Quantitative parameters are displayed as color maps on a pixel-by-pixel basis (Fig. 1A). Main quantitative parameters automatically generated by software include blood flow (BF, Fig. 1B) per unit volume or mass of tissue, which represents the rate of delivery of oxygen and nutrients; tumor relative blood volume (BV, Fig. 1C), which represents the volume of the functioning vascular network; mean transit time(MTT, Fig. 1D) is defined as the time that a contrast agent takes to go through the mass of tissue, from entry to exit; permeability surface area product (PS, Fig. 1E) , which describes the rate of contrast leakage into the extracellular space [16-20]; and some other quantitative parameters, such as time of arrival (IRF TO, Fig. 1F), maximum slope of increase (MSI, Fig. 1G) and positive enhancement integral (PEI, Fig. 1H), time to peak (TTP, Fig. 1I) etc. In general, perfusion measurements are temporally and spatially variable due to the underlying physiology and intratumor heterogeneity. The validity of these measured parameters have been widely verified in animal and in human studies [17, 20, 21].

\section{Clinical Applications}

CT perfusion in oncology is based on the principle that that the microvascular changes of tumor angiogenesis is reflected by the increased tumor vascularization [17]. Since the first application in the evaluation of solitary pulmonary nodules [14], CT perfusion of lung nodules have evolved for lesion characterization, cancer staging, predicting patient outcome or response to therapy, assessment of relapse and tumor resistance to treatments, and pharmacodynamic analysis for cancer drug development.

\section{Lesion Characterization}

Dynamic CT perfusion with a single-detector or MDCT scanner had been utilized for differentiating cancers from benign nodules over the last few decades. Several structural abnormalities of tumor vessels are characteristic $[3,22,23]$ and lead to pathophysiologic changes within the tumor, including the increase of capillary permeability and tumor perfusion, which is the distinctive features suggesting malignancy. 

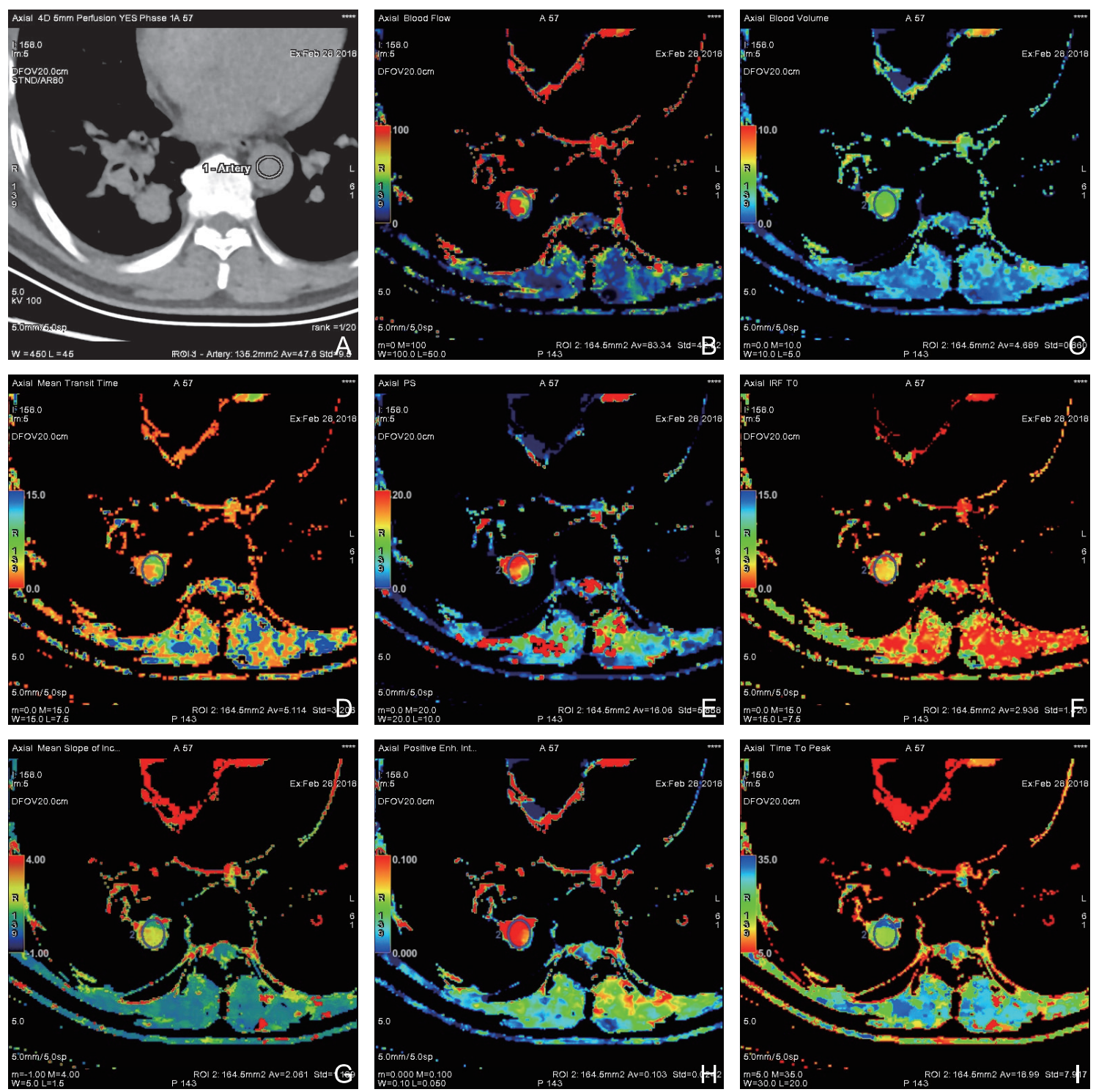

Figure 1 A-I, A 60 years old man with pulmonary nodule. B, located in the right lower lobe. CT perfusion parameters BF (B), BV (C), MTT (D), PS (E), IRF TO (F), MSI (G), PEI (H), TTP (I) values derived from the perfusion maps by perfusion software.

Significant differences were seen when CT perfusion parameters between normal tissue and tumors, benign and malignant lesions were compared, although there is overlap between malignant and inflammatory pulmonary lesions $[15,24,25]$. In general, higher perfusion parameters have been reported in patients with lung tumors, although the angiogenic phenotype is diverse between different type of lung tumor and in the same type of lung cancer. According to current reports, some studies showed that CT perfusion parameter such as BF, BV and PS values are higher in malignant pulmonary nodules than benign nodules [26-28] (Fig. 2A D vs. Fig. 3A D), except for some individual value that fails to achieve statistical difference, such as PS value in Sitartchouk's study [26], BF value in Shu's study [28].These investigators have examined the capability of dynamic CT perfusion with 64-MDCT for the differentiation of malignant from benign nodules, and reported that the sensitivity, specificity, and accuracy were $79 \%-91 \%, 81 \%-96 \%$, and $80 \%-96 \%$, respectively [26-28]. CT perfusion parameters combined with routine $\mathrm{CT}$ may be more sensitive and accurate for differentiating malignancies from benign lung nodules than conventional $\mathrm{CT}$ alone and allows more confidence and constancy.

For further identifying different characteristics of lung nodules, investigators studied CT perfusion parameters among malignant, benign and active inflammatory SPNs and found that mean perfusion, $\mathrm{BV}$ and peak enhancement intensity (PEI) of benign SPNs were significantly lower than those for malignant 
nodules (lung cancer) (Fig. 2C, E vs Fig. 3C, E) and active infections, but the differences were not statistically significant between malignant tumors (lung cancer) and active infections $[15,24,25,29]$.
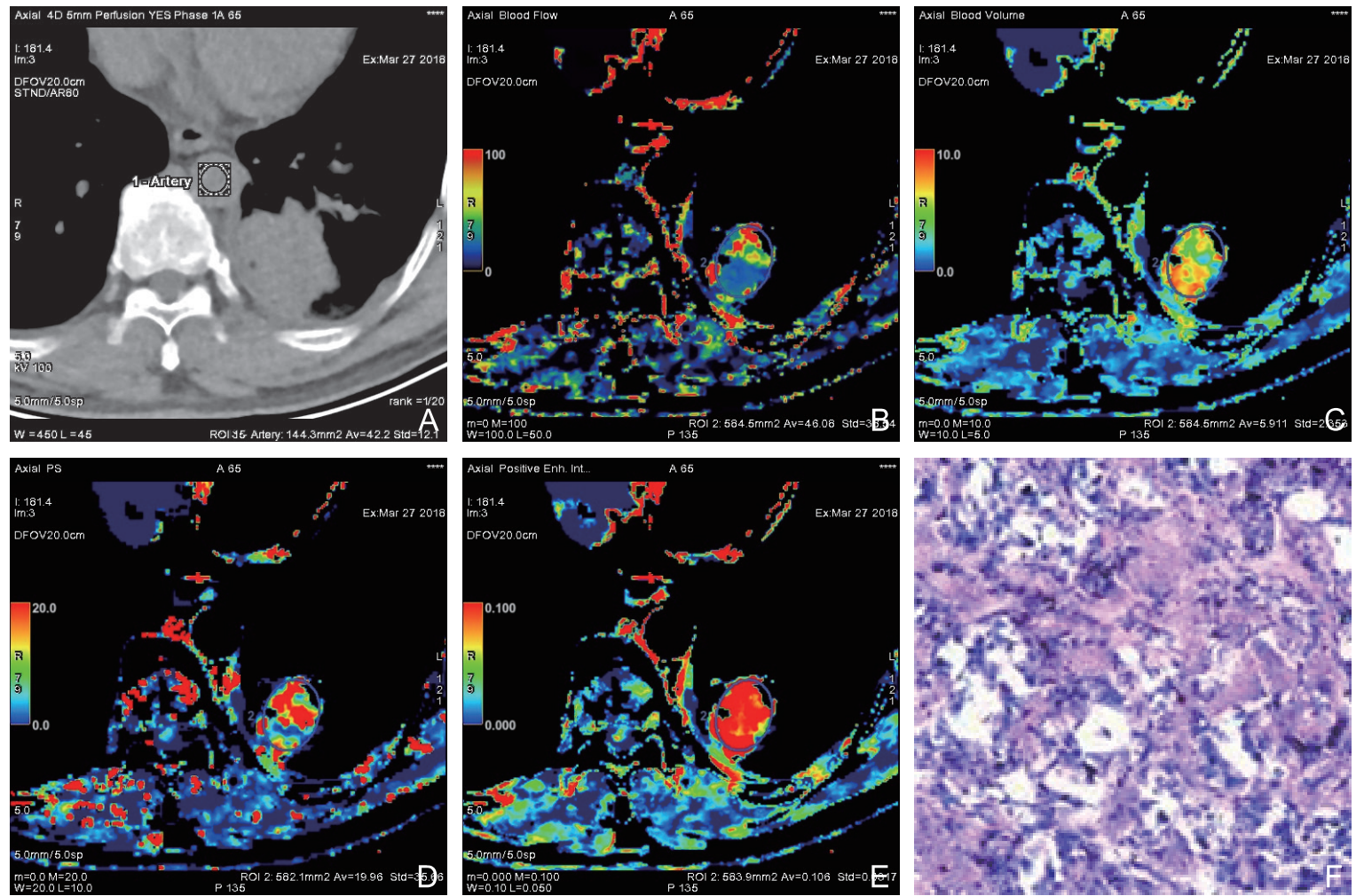

Figure 2 A 53 years old man with pulmonary nodule. A, located in the left lower lobe. CT perfusion parameters BF (B), BV (C), PS(D) and PEI (E) in the nodule (A) of non-small cell lung cancer proved by pathological specimen (F).
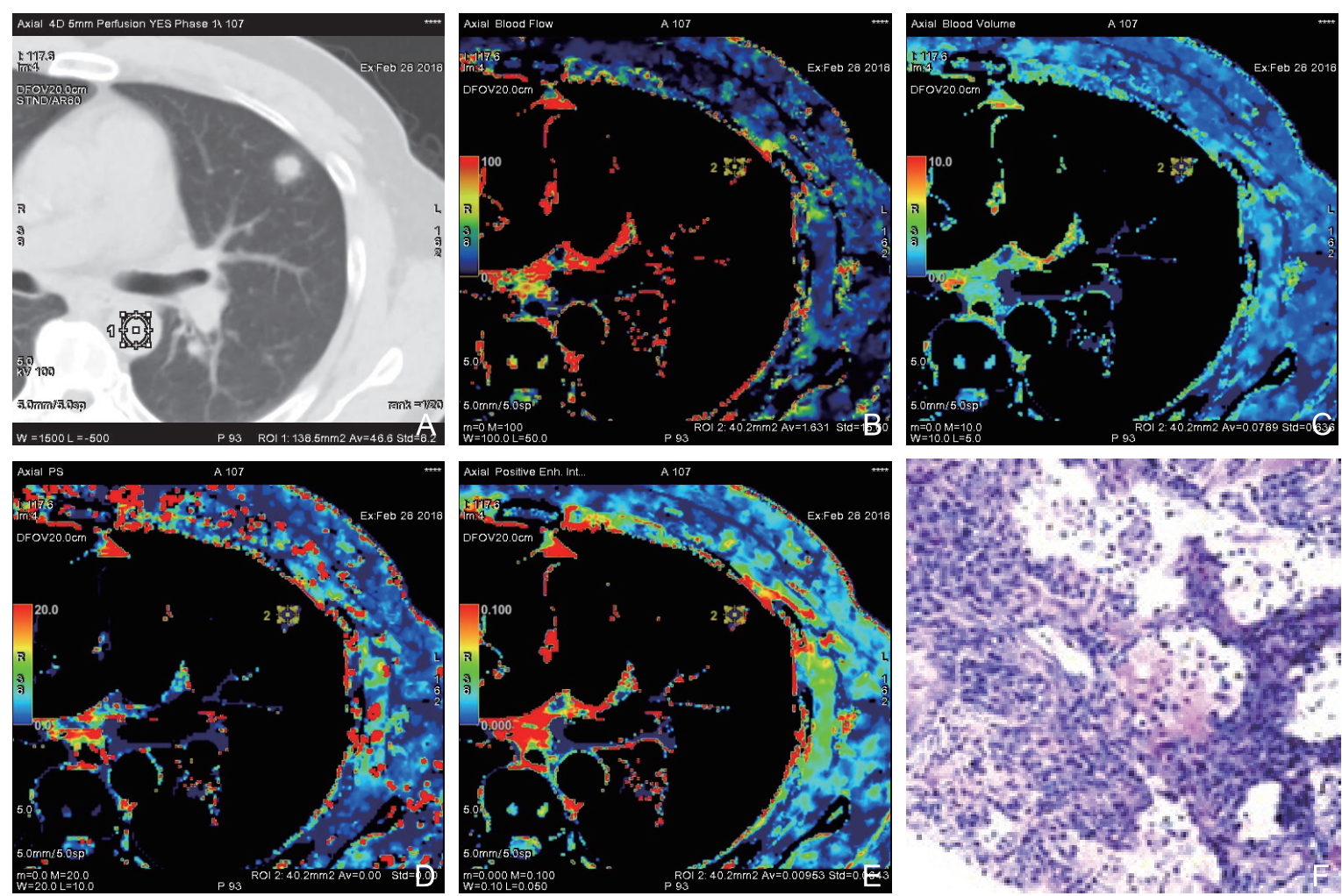

Figure 3 A 61-year-old man with pulmonary nodule. A, located in the left upper lobe. CT perfusion parameters BF (B), BV (C), PS (D) and PEI (E) in the nodule (A) of inflammatory nodule proved by pathological specimen (F). BF, BV, PS and PEI in the previous malignant nodule (Figure. 2A) were higher than the latter benign nodule (Figure. $3 \mathrm{~A})$. 
Besides, the findings of $\mathrm{CT}$ perfusion values were used for the differentiation of cavitary mass. Perfusion maps [volume, washout, peak and time-to-peak (TTP)] were derived on the pixel-by-pixel basis from the timeto-density curve. Pyogenic cavities showed weak washout and slow TTP (69.6\%). On the contrary, malignant cavities showed strong washout $(73.3 \%)$. Tuberculous cavities showed low perfusions in the volume and peak maps (66.7\%). The performance of the combination of conventional $\mathrm{CT}$ and perfusion $\mathrm{CT}$ was better than conventional CT alone [30].

Only one study reported the usefulness of CT perfusion in the differentiation of different type of lung cancer. Their result showed CT perfusion parameters were the most significant for adenocarcinoma, followed by small cell carcinoma, large cell carcinoma and squamous cell carcinoma, but no statistical differences were found $(P>0.05)$ [31]. While iodine concentration, spectral curve slope and $\mathrm{CT}$ values between squamous carcinoma and adenocarcinoma at $40 \mathrm{keV}$ of high-definition Computed Tomographic Gemstone Spectral Imaging were significantly different [32]. However, CT perfusion characteristics are helpful to evaluate the histology differentiation of lung adenocarcinoma. The cancers which have the drastical decrease of perfusion parameters tend to be of lower grade of differentiation. BF better correlated with differentiation grade, compared to BV and PEI [32].

\section{Staging}

Perfusion may also be useful in tumor staging. Ippolito et al [31] presented evidence that dynamic $\mathrm{CT}$ perfusion is suitable for discriminating $\mathrm{T} 1$ and T2 lung cancer (i.e. T1b vs. T2a) according to the TNM classification. There were significant perfusion differences (lower blood volumes and higher mean transit time) between tumors with diameter $>30 \mathrm{~mm}$ and tumors with diameter $<30 \mathrm{~mm}$. BV was significantly higher in lesions with the diameter less than $30 \mathrm{~mm}$, while BF showed a tendency to be higher in smaller lesions. In contrast, MTT was significantly higher in lesions with the diameter $\geqq 30 \mathrm{~mm}$. However, Huellner, et al [33] suggested that CT perfusion showed little additional information for the initial staging compared with standard FDG-PET/CT. The tumors in patients displaying positive regional lymph node metastases on PET had significantly lower BF and BV than the tumors with PET-negative nodes. BV was significantly different, depending on the $\mathrm{N}$ status, with higher values in N0 and N2 tumors, and lower values in N1 and N3 tumors $(P=0.005)$. However, the CT perfusion values were not significantly different among $\mathrm{N}$ stages. CT perfusion values were not different among the tumors with different size and location. Therefore, it may be important to further investigate the current inconsistence of results regarding the applications of $\mathrm{CT}$ perfusion for staging lung cancers.

\section{Relationship Between Different CT Perfusion}

\section{Markers}

In terms of a predictive values of different imaging biomarker, CT perfusion studies of lung tumors suggest that the perfusion parameter are reflective of histologic markers, such as VEGF expression, microvessel density (MVD) and maximal standard uptake value (SUVmax) of FDG-PET/CT. Li et al assessed the correlation between the perfusion parameters and lung tumor angiogenesis and fund that perfusion parameters, such as PEI and BV are positively correlated with extent of MVD [25]. Shu et al found SPNs with high expression VEGF showed significantly higher CT perfusion values, including higher BV, PS, BF, MTT, PEI and TTP than those of the SPNs with lower VEGF expression. And in the cancer lesions with high VEGF expression, MVD was found to be positively correlated with $\mathrm{BF}, \mathrm{BV}$, and PS of malignant SPNs. MVD was not correlated with $\mathrm{BF}, \mathrm{BV}$, and PS in the three groups of SPNs with low VEGF expression [28]. However, the study by Xiong et al suggests that the imaging characteristics derived from CT perfusion are pathologically based on the density of immature microvessels rather than microvessel density (MVD) in lung adenocarcinoma [32]. Huellner et al found there was no significant correlation between CT perfusion parameters and MVD [33]. A trend of higher values of SUVmax was shown in the cancer lesions with the diameter $\geqslant 30 \mathrm{~mm}$. Furthermore, a significant inverse relationship between BF and SUVmax was shown in lesions with the diameter more than $30 \mathrm{~mm}$. On the contrary, a significant linear relationship between MTT and SUVmax was shown in the same tumors [31]. Therefore, it is desirable to explore the relationship between different markers such as CT perfusion parameters, VEGF expression, MVD and SUVmax.

\section{Prognostic Biomarker and Response Prediction}

The response of lung cancers to therapies are usually evaluated by morphological criteria such as RECIST 1.1 on CT or MRI. However, those criteria are insufficient because they are based on tumoral dimensional changes and other morphologic features were not taken into account such as cell density, functional or metabolic changes that may occur following conventional, targeted chemotherapy, radiation therapy, and immunotherapy. 
The chemotherapies with different agents (antiangiogenic drugs, vascular disrupting agents and immunotherapy), radiotherapy and interventional procedures, such as radiofrequency ablation, will alter tumor vascularization. A long-term effect of treatment is demonstrated by the decrease of CT perfusion parameters, but in the short term, there is variable vascular effect related to the therapeutic mechanism of action. Several studies revealed that CT perfusion parameters is useful in the assessment of patients with lung tumors undergoing different therapies $[13,18,21$, 24, 34-42].

For patients with lung cancer treated with standard chemotherapy, tumor shrinkage was seen as a sign of the cytotoxic effect. Its vascular effect is thought to reflect the loss of angiogenic cytokine support following cell death [43]. Kiessling et al [24] described a decrease of tumor perfusion in non-small cell lung cancers (NSCLC) after two cycles of conventional chemotherapy. While Fraioli et al [40] showed the decrease of perfusion parameters such as BF and PS after platinum-based chemotherapy plus bevacizumab in non-squamous carcinoma and platinum-based doublets for squamous carcinoma.

Angiogenesis inhibitors have different targets and their anti-vascular effects vary depending on the mechanism of action of the drug under investigation. An initial effect may be a decrease in vascular permeability and interstitial fluid pressure, following a transient increase of tumor blood flow due to the normalization of function of the vasculature [44]. Consequently, regional tissue perfusion is gradually reduced with subsequent pruning of the vasculature. The effects of anti-angiogenic agents (EGFR inhibitors) have also been investigated $[38,41]$ in patients with NSCLC. Mean tumor perfusion decreased significantly after 6-week treatment. CT perfusion was lower in RECIST responders versus non-responders at week 3 and week 6 respectively. Another study showed dynamic CT perfusion can used to early evaluate targeted therapy efficacy 7 days post-treatment and reduced blood perfusion suggests tumor sensitivity to therapy and treatment effectiveness [45].

With radiotherapy, the acute effects are related to an initial inflammatory effect; the change in permeability is related to microvascular damage, which can lead to tumor shrinkage [46]. A significant decrease in BF, BV PS and increase in MTT of CT perfusion parameters were affirmed in NSCLC patients following two cycles of chemo-radiotherapy [34].Furthermore, tumor perfusion of RECIST responders is significantly higher than non-responders after chemo-radiotherapy, as well as the feasible mean overall survival (OS) and progression free survival(PFS) determined with interaction of chemotherapy and radiotherapy[13,42].

\section{Limitations and Development}

Dynamic CT perfusion studies are increasingly used in medical care and clinical applications in lung tumors. What CT perfusion parameters are optimal for diagnosis, prognosis prediction, therapy planning and/or treatment monitoring? The primary objective of optimizing CT perfusion is to set up the examination protocol in such a way that these microcirculatory parameters can be achieved with sufficiently high accuracy (low bias or systematic error) and precision (low variance or statistical error) by tracer kinetic analysis from a minimum set of serial CT scans acquired at the least possible dose.

Basically, lung tumors receive their blood supply from both the arterial and pulmonary venous systems. Incorporation of such dual input functions in the physiologic modeling and data acquisition is extremely challenging and is not widely implemented. Mathematical models have a significant influence on the assessment of early response to treatments, evaluation of disease progression and overall survival using dynamic CT perfusion for NSCLC treated with chemoradiotherapy. Moreover, the dual-input maximum slope model rather than the single-input maximum slope and Patlak Plot model should be used for the evaluation of perfusion parameters [13].

Secondly, respiratory motions are potential factors hampering the reproducibility of perfusion parameters as well as the absolute values of CT-measured parameters [37]. Serial CT scanning of the chest is best performed for total acquisition time of 30-50s of first-pass perfusion during breath-hold, which is necessary to ensure the consistent drawing of ROIs in tumors.

Nevertheless, the radiation dose resulting from one or even a few $\mathrm{CT}$ perfusion examinations has been significantly reduced and a slightly higher radiation dose may be acceptable and feasible for patients with advanced lung cancer and poor prognosis [47].

In addition, it is important to understand the inherent measurement variability in evaluation of lung cancers using perfusion CT. With the increasing tendency to use combination anticancer therapies, the assessment of the anti-angiogenesis effect of every single therapy in tumors may be difficult. Up to now, the optimal time to perform $\mathrm{CT}$ perfusion after the start of an antitumor treatment varies and may influence therapeutic evaluation.

\section{Conclusion}

In summary, parameters derived from CT perfusion parameters of lung nodules for the diagnosis, differential diagnosis, evaluating the correlations between these 
parameters and clinical markers, is important in understanding lung tumor aggressiveness, and plays a role in indicating prognosis and in therapeutic monitoring of lung carcinoma following chemotherapy, radiotherapy and other therapies. CT perfusion is more specific and accurate than dynamic MR imaging and FDG PET/ $\mathrm{CT}$ in the diagnosis of solitary pulmonary nodules and the therapeutic effect of lung tumors in routine clinical practice [11-14,42,47]. Different patient cohorts receiving inconsistent imaging protocols and disparate treatment regimens in these studies highlight the need for the standardization and validation to push on widespread clinical application, for obtaining the required and valuable medical information, better economic and societal value.

\section{Conflict of Interest}

The authors have no conflict of interest to declare.

\section{References}

[1] Hanahan D, Weinberg RA. Hallmarks of cancer: the next generation. Cell 2011;14:646-74.

[2] Folkman J. Tumor angiogenesis: therapeutic implications. $N$ Engl $J$ Med 1971;285:1182-6.

[3] Janning M, Loges S. Anti-Angiogenics: Their Value in Lung Cancer Therapy. Oncol Res Treat 2018;41:172-180.

[4] Gummadi S, Eisenbrey J, Li JZ, Li ZJ, Forsberg F, Lyshchik A, et al. Advances in modern clinical ultrasound. AUDT 2018;2:51-63.

[5] Chang YC, Yu CJ, Chen CM, Hu FC, Hsu HH, Tseng WY, et al. Dynamic contrast-enhanced MRI in advanced nonsmall-cell lung cancer patients treated with first-line bevacizumab, gemcitabine, and cisplatin. J Magn Reson Imaging 2012;36:387-96.

[6] Choyke PL, Dwyer AJ, Knopp MV. Functional tumor imaging with dynamic contrast-enhanced magnetic resonance imaging. J Magn Reson Imaging 2003;17:509-20.

[7] Pishko GL, Muldoon LL, Pagel MA, Schwartz DL, Neuwelt EA. Vascular endothelial growth factor blockade alters magnetic resonance imaging biomarkers of vascular function and decreases barrier permeability in a rat model of lung cancer brain metastasis. Fluids Barriers CNS 2015;12:5.

[8] Koenigkam-Santos M, Optazaite E, Sommer G, Safi S, Heussel CP, Kauczor HU, et al. Contrast-enhanced magnetic resonance imaging of pulmonary lesions: description of a technique aiming clinical practice. Eur J Radiol 2015;84:185-192.

[9] Broncano J, Luna A, Sánchez-González J, Alvarez-Kindelan A, Bhalla S. Functional MR Imaging in Chest Malignancies. Magn Reson Imaging Clin N Am 2016;24:135-155.

[10] Mirsadraee S, van Beek EJ. Functional imaging: computed tomography and MRI. Clin Chest Med 2015;36:349-63.

[11] Ohno Y, Nishio M, Koyama H, Fujisawa Y, Yoshikawa T, Matsumoto $\mathrm{S}$, et al. Comparison of quantitatively analyzed dynamic areadetector CT using various mathematic methods with FDG PET/CT in management of solitary pulmonary nodules. AJR Am J Roentgenol 2013;200:W593-602.

[12] Ohno Y, Nishio M, Koyama H, Seki S, Tsubakimoto M, Fujisawa $\mathrm{Y}$, et al. Solitary pulmonary nodules: Comparison of dynamic firstpass contrast-enhanced perfusion area-detector $\mathrm{CT}$, dynamic first- pass contrast-enhanced MR imaging, and FDG PET/CT. Radiology 2015;274:563-75.

[13] Ohno Y, Koyama H, Fujisawa Y, Yoshikawa T, Seki S, Sugihara N, et al. Dynamic contrast-enhanced perfusion area detector CT for nonsmall cell lung cancer patients: Influence of mathematical models on early prediction capabilities for treatment response and recurrence after chemoradiotherapy. Eur J Radiol 2016;85:176-186.

[14] Ohno Y, Koyama H, Matsumoto K, Onishi Y, Takenaka D, Fujisawa $\mathrm{Y}$, et al. Differentiation of malignant and benign pulmonary nodules with quantitative first-pass 320-detector row perfusion CT versus FDG PET/CT. Radiology 2011;258:599-609.

[15] Chae EJ, Song JW, Seo JB, Krauss B, Jang YM, Song KS. Clinical utility of dual-energy $\mathrm{CT}$ in the evaluation of solitary pulmonary nodules: initial experience. Radiology 2008;249:671-81.

[16] Purdie TG, Henderson E, Lee TY. Functional CT imaging of angiogenesis in rabbit VX2 soft-tissue tumour. Phys Med Biol 2001;46:3161-75.

[17] Miles KA. Perfusion CT for the assessment of tumour vascularity: which protocol? Br J Radiol 2003;76 Spec No 1:S36-42.

[18] Petralia G, Bonello L, Viotti S, Preda L, d'Andrea G, Bellomi M. CT perfusion in oncology: how to do it. Cancer Imaging 2010;10:8-19.

[19] Kambadakone AR, Sahani DV. Body perfusion CT: technique, clinical applications, and advances. Radiol Clin North Am 2009;47:161-78.

[20] Miles KA, Lee TY, Goh V, Klotz E, Cuenod C, Bisdas S, et al. Current status and guidelines for the assessment of tumour vascular support with dynamic contrast-enhanced computed tomography. Eur Radiol 2012;22:1430-41.

[21] Tacelli N, Remy-Jardin M, Copin MC, Scherpereel A, Mensier E, Jaillard S, et al. Assessment of non-small cell lung cancer perfusion: pathologic-CT correlation in 15 patients. Radiology 2010;257:86371.

[22] Ebos JM, Kerbel RS. Antiangiogenic therapy: impact on invasion, disease progression, and metastasis. Nat Rev Clin Oncol 2011;8:21021.

[23] Figueiras RG, Padhani AR, Goh VJ, Vilanova JC, González SB, Martín CV, et al. Novel oncologic drugs: what they do and how they affect images. Radiographics 2011;31:2059-91.

[24] Kiessling F, Boese J, Corvinus C, Ederle JR, Zuna I, Schoenberg SO, et al. Perfusion $\mathrm{CT}$ in patients with advanced bronchial carcinomas: a novel chance for characterization and treatment monitoring? Eur Radiol 2004;14:1226-33.

[25] Li Y, Yang ZG, Chen TW, Yu JQ, Sun JY, Chen HJ. First-pass perfusion imaging of solitary pulmonary nodules with 64-detector row CT: comparison of perfusion parameters of malignant and benign lesions. Br J Radiol 2010;83:785-90.

[26] Sitartchouk I, Roberts HC, Pereira AM, Bayanati H, Waddell T, Roberts TP. Computed tomography perfusion using first pass methods for lung nodule characterization. Invest Radiol 2008;43:34958.

[27] Shan F, Zhang Z, Xing W, Qiu J, Yang S, Wang J, et al. Differentiation between malignant and benign solitary pulmonary nodules: use of volume first-pass perfusion and combined with routine computed tomography. Eur J Radiol 2012;81:3598-605.

[28] Shu SJ, Liu BL, Jiang HJ. Optimization of the scanning technique and diagnosis of pulmonary nodules with first-pass 64-detector-row perfusion VCT. Clin Imaging 2013;37:256-64.

[29] Ma SH, Le HB, Jia BH, Wang ZX, Xiao ZW, Cheng XL, et al. Peripheral pulmonary nodules: relationship between multi-slice spiral CT perfusion imaging and tumor angiogenesis and VEGF expression. BMC Cancer 2008;8:186.

[30] Lee YH, Kwon W, Kim MS, Kim YJ, Lee MS, Yong SJ, et al. Lung 
perfusion CT: the differentiation of cavitary mass. Eur J Radiol 2010;73:59-65.

[31] Wang G, Zhang C, Li M, Deng K, Li W. Preliminary application of high-definition computed tomographic Gemstone Spectral Imaging in lung cancer. J Comput Assist Tomogr 2014;38:77-81.

[32] Xiong Z, Liu JK, Hu CP, Zhou H, Zhou ML, Chen W. Role of immature microvessels in assessing the relationship between CT perfusion characteristics and differentiation grade in lung cancer. Arch Med Res 2010;41:611-7.

[33] Huellner MW, Collen TD, Gut P, Winterhalder R, Pauli C, Diebold J, et al. Multiparametric PET/CT-perfusion does not add significant additional information for initial staging in lung cancer compared with standard PET/CT. EJNMMI Res 2014;4:6.

[34] Wang J, Wu N, Cham MD, Song Y. Tumor response in patients with advanced non-small cell lung cancer: perfusion $\mathrm{CT}$ evaluation of chemotherapy and radiation therapy. AJR Am J Roentgenol 2009;193:1090-6.

[35] Hegenscheid K, Behrendt N, Rosenberg C, Kuehn JP, Ewert R, Hosten $\mathrm{N}$, et al. Assessing early vascular changes and treatment response after laser-induced thermotherapy of pulmonary metastases with perfusion CT: initial experience. AJR Am J Roentgenol 2010;194:1116-23.

[36] Fraioli F, Vetere S, Anile M, Venuta F. Computed tomography perfusion: a new method to evaluate response to therapy in lung cancer. J Thorac Oncol 2011;6:1599-600.

[37] Ng CS, Chandler AG, Wei W, Anderson EF, Herron DH, Charnsangavej $\mathrm{C}$, et al. Reproducibility of perfusion parameters obtained from perfusion CT in lung tumors. AJR Am J Roentgenol 2011;197:113-21.

[38] Lind JS, Meijerink MR, Dingemans AM, van Kuijk C, Ollers MC, de Ruysscher D, et al. Dynamic contrast-enhanced CT in patients treated with sorafenib and erlotinib for non-small cell lung cancer: a new method of monitoring treatment? Eur Radiol 2010;20:2890-8.

[39] Bellomi M, Viotti S, Preda L, D'Andrea G, Bonello L, Petralia G.
Perfusion CT in solid body-tumours. Part II: Clinical applications and future development. Radiol Med 2010;115:858-74.

[40] Fraioli F, Anzidei M, Zaccagna F, Mennini ML, Serra G, Gori B, et al. Whole-tumor perfusion $\mathrm{CT}$ in patients with advanced lung adenocarcinoma treated with conventional and antiangiogenetic chemotherapy: initial experience. Radiology 2011;259:574-82.

[41] Fraioli F, Anzidei M, Serra G, Liberali S, Fiorelli A, Zaccagna F, et al. Whole-tumour CT-perfusion of unresectable lung cancer for the monitoring of anti-angiogenetic chemotherapy effects. $\mathrm{Br} J$ Radiol 2013;86(1029):20120174.

[42] Ohno Y, Fujisawa Y, Koyama H, Kishida Y, Seki S, Sugihara N, et al. Dynamic contrast-enhanced perfusion area-detector CT assessed with various mathematical models: Its capability for therapeutic outcome prediction for non-small cell lung cancer patients with chemoradiotherapy as compared with that of FDG-PET/CT. Eur $J$ Radiol 2017;86:83-91.

[43] Lissoni P, Fugamalli E, Malugani F, Ardizzoia A, Secondino S, Tancini G, et al. Chemotherapy and angiogenesis in advanced cancer: vascular endothelial growth factor (VEGF) decline as predictor of disease control during taxol therapy in metastatic breast cancer. Int $J$ Biol Markers 2000;15:308-11.

[44] Jain RK. Normalization of tumor vasculature: an emerging concept in antiangiogenic therapy. Science 2005;307:58-62.

[45] Qiao PG, Zhang HT, Zhou J, Li M, Ma JL, Tian N, et al. Early evaluation of targeted therapy effectiveness in non-small cell lung cancer by dynamic contrast-enhanced CT. Clin Transl Oncol 2016;18:47-57.

[46] Garcia-Barros M, Paris F, Cordon-Cardo C, Lyden D, Rafii S, Haimovitz-Friedman A, Fuks Z, et al. Tumor response to radiotherapy regulated by endothelial cell apoptosis. Science 2003;300:1155-9.

[47] Brix G, Lechel U, Nekolla E, Griebel J, Becker C. Radiation protection issues in dynamic contrast-enhanced (perfusion) computed tomography. Eur J Radiol 2015;84:2347-58. 